\title{
Desastres naturales y prevención de enfermedades
}

\section{Natural disasters and prevention of communicable diseases}

Correspondencia

Ciro Maguiña Vargas

ciro.maguina@upch.pe

Recibido: 12/03/2017

Aprobado: 15/03/2017

Citar como: Maguiña C, Astuvilca J. Desastres naturales y prevención de enfermedades. Acta Med Peru. 2017;34(1):3-5

\author{
Ciro Maguiña Vargas ${ }^{1,2,3, a, b}$, Juan Astuvilca Cupe ${ }^{4,5, c, d}$ \\ 1 Vicerrectorado de Investigación, Universidad Peruana Cayetano Heredia. Lima, Perú. \\ 2 Instituto de Medicina Tropical "Alexander von Humboldt", Universidad Peruana Cayetano Heredia. Lima, Perú. \\ 3 Departamento de Enfermedades Infecciosas, Tropicales y Dermatológicas, Hospital Nacional Cayetano Heredia. Lima, Perú. \\ 4 Facultad de Medicina, Universidad Científica del Sur. Lima, Perú. \\ 5 Colegio Médico del Perú. \\ a Vicerrector; b Médico Infectólogo, Tropicalista, Dermatólogo; c Docente; d Médico Epidemiólogo
}

El Perú, debido a su variada y diversa geografía, es considerado uno de los 12 países del mundo con mayor biodiversidad; en contraste, presenta una serie notable de carencias en agua potable, alcantarillado, vivienda, y otras necesidades básicas que hacen que, según el estudio Tyndall Center (2003), se encuentre dentro de los 10 países más vulnerables a los eventos climáticos, solo antecedido por Honduras, Bangladesh y Venezuela; por ello, es afectado de manera importante por los efectos del cambio climático que producen el calentamiento de la temperatura del mar, huaycos, inundaciones, etc.; esto a su vez, afecta de manera significativa a la salud de las poblaciones ${ }^{[1]}$.

Los antiguos peruanos adoraban a la naturaleza y la respetaban, por ello nunca se atrevieron a construir sus templos en cauces y quebradas; así son ejemplos de eternidad, el antiguo Caral con 5000 años de antigüedad en el Valle de Supe ubicado a 30 metros del cauce del río, Chavín ubicada a $3200 \mathrm{~m}$ de altitud que resistió siempre al clima y los terremotos, Machu Picchu a $2400 \mathrm{~m}$ de altitud, Kuelap a 3000 m de altitud, Huanucopampa a $3200 \mathrm{~m}$ de altitud y otras como Warcahuamachuco en la Libertad. En la costa destacaban Pachacámac, Paramonga, Chanquillo, Incahuasi muy cerca al valle de Lunahuaná que luce activo e imponente, y las huacas del Sol y la Luna ubicadas en cerros más altos que el río Moche. En cambio, otras ruinas como Chan Chan, Túcume, Sipán, Sicán, Chotuna, Huaca $\mathrm{Cao}$, que en sus tiempos fueron templos sagrados y hasta hoy dejan ver sus imponentes restos, fueron castigados por los sucesivos Fenómenos del Niño y sequías, y desaparecieron como civilización. Pero en la castigada Piura hubo una huaca sagrada que resistió: Narihualá de los míticos Tallanes, que gracias a su altitud, heroicamente resistió a la terrible inundación y fue refugio de los pobres de Piura en este último desastre natural ${ }^{[2]}$.

De acuerdo al informe "Riesgo potencial epidémico, frente a los efectos del Fenómeno El Niño" desarrollado por la Dirección General de Epidemiologia del Ministerio de Salud del Perú, los sistemas de agua potable y alcantarillado serán los primeros en colapsar, especialmente en la primera semana, produciendo un incremento en el número de casos con enfermedades diarreicas agudas. Se señala también que la exposición de las personas a áreas anegadas (por lluvias o inundaciones) y la concentración de partículas de polvo en el aire (por remoción de escombros), incrementarían los casos de infecciosas dérmicas y oculares (conjuntivitis entre las más frecuentes), además de infecciones respiratorias. De igual forma, el grupo poblacional más afectado por las inundaciones generalmente es el infantil, siendo las patologías de la piel más frecuentes: excoriaciones o heridas superficiales, dermatitis alérgicas, piodermitis y micosis ${ }^{[1,3]}$. 
De todos los fenómenos estacionales, el de El Niño representa el fenómeno que más ha afectado e impactado en la variabilidad climática regional ${ }^{[3,4]}$. Así, en el Perú, el evento de 1998 causó aproximadamente 340 muertes, 300000 damnificados, destruyó 40000 hectáreas de cultivo, y la afectación de sectores como la pesca ${ }^{[3,5,6]}$.

En las semanas de febrero a marzo del 2017 hemos visto una vez más que el cambio climático ha producido en el norte del Perú intensas lluvias, y ello a su vez ha causado grandes desastres en muchas regiones del país que han hecho colapsar los sistemas de agua potable y desagüe en ciudades como Piura y Huarmey, y en otras que también han sido afectadas seriamente dentro de los departamentos de Tumbes, Lambayeque, La Libertad y Ancash.

Desde hace muchas décadas se conoce que el calentamiento del agua del mar trastorna el equilibrio ecológico del planeta, lo que a su vez determina en el humano la aparición o reemergencia de diversas dolencias, algunas de las cuales se transmiten por el agua contaminada y otras por los vectores, muchos de ellos transmisores de enfermedades metaxénicas (malaria, dengue, enfermedad de Carrión, leishmaniasis, chikungunya, etc.). Todo ello está causando en las zonas afectadas, el incremento de manera notable de enfermedades como diarreas agudas, infecciones respiratorias agudas, infecciones de piel, entre otros problemas.

Respecto al agua contaminada, y dependiendo de la zona, se pueden presentar epidemias de cólera, fiebre tifoidea, salmonelosis, leptospirosis, cyclosporidiasis, criptosporidiosis y hepatitis $A$. En relación a los vectores, se conoce que el incremento en la temperatura favorece la reproducción de los mosquitos y así, en corto tiempo, podría haber más mosquitos transmisores $y$, por tanto, más enfermos.

Durante el Fenómeno de El Niño en 1998, se manifestó claramente un incremento en la incidencia de algunas condiciones dermatológicas tales como las tiñas, la pitiriasis versicolor, la miliaria y la foliculitis. El estudio de Bravo Sosa y Bravo Succio (2001) describió un aumento importante en el número de casos de miliarias atendidas en el Hospital Nacional Cayetano Heredia, sobre todo en la población pediátrica. Sólo cuatro casos se atendieron en el verano de 1997 (sin fenómeno de El Niño), mientras que en el año de 1998 (con fenómeno de El Niño) los casos llegaron a $97^{[7]}$. Este aumento en el número de miliarias no queda solo como un factor numérico, pues, aunque el estudio mencionado no lo determinó con exactitud, muchos de estos casos de miliaria se complicaron y demandaron muchas más horas de atención. Muchas de estas miliarias, se suelen sobreinfectar y dar origen a estreptodermias y estafilodermias ${ }^{[7,8]}$.

Se ha visto además que las peligrosas y pequeñas arañas marrones, como la especie Loxosceles laeta, que viven en las casas especialmente de la costa peruana, debido al incremento de la temperatura (mayor reproducción) y la afectación de las casas, producen más ataques al humano, siendo el cuadro viscerohemolítico más frecuente, el cual puede ser letal si no es tratado a tiempo; por ello se deben tomar medidas preventivas frente a este tipo de accidentes. También en muchas áreas de la costa y selva, las serpientes venenosas de la especie Bothrops pictus (jergón de la costa) presentes en La Libertad, Lima e Ica, o el Bothrops barnetti (macanche) en Piura, Cajamarca y Lambayeque, son las que causan una mayor incidencia de mordeduras por la afectación y vulnerabilidad de las viviendas. A todo ello se suma el grave problema de la afectación de la salud mental al inicio, durante y posterior al desastre natural.

Los cambios ecológicos también favorecen la reproducción de insectos vectores y roedores, así las enfermedades zoonóticas como la leptospirosis y la peste, podrían aparecer entre 1 a 4 semanas de ocurrido el evento; y las ocasionadas por vectores como el dengue y la malaria, a partir de las 4 semanas ${ }^{[9-11]}$.

Respecto a la actual distribución de estas enfermedades en el Perú: la malaria, dengue y chikungunya se distribuye generalmente en la región norte y nororiental, en selva alta y baja; la peste bubónica, en la costa y sierra norte; rabia silvestre y urbana, en la sierra sur y selva; la leptospira en el norte del país y nororiente, en selva alta y baja; y la enfermedad de Carrión y la leishmaniasis en los valles interandinos y selva alta.

Para el control y prevención de las diversas patologías señaladas se deberían realizar las siguientes acciones: 1) evaluar rápidamente los riesgos, 2) seleccionar y planificar los sitios de refugio, 3) garantizar el empleo de agua segura, 4) saneamiento básico (disposición de basura y excretas), 5) higiene y alimentos seguros, 6) realizar intervenciones para el control de vectores, y 7) proporcionar servicios de salud esenciales con instalación de laboratorio básico.

Además, como país se debe organizar una respuesta basada en "sistemas de salud" y en "servicios de salud esenciales". Referente a la respuesta como sistema de salud, se incluyen seis componentes primordiales: 1 ) recursos humanos (capacitados y competentes), 2) medicamentos y suministros (acceso esencial continuo), 3) financiación de los servicios de salud (acceso a atención primaria gratuita mientras dura el desastre), 4) gestión de información en salud (análisis e interpretación de datos para establecer planes de emergencia y sistemas de vigilancia epidemiológica), 5) prestación de servicios (servicios estandarizados y con guías para el diagnóstico y tratamiento de daños más comunes) y 6) liderazgo y coordinación (acceso a servicios de salud coordinados entre las diferentes organizaciones y sectores) ${ }^{[12]}$.

Con las lecciones aprendidas en todos estos años, debemos prepararnos con un enfoque país basado en un sistema de salud unificado, ya que como consecuencia del cambio climático en el planeta, es probable que estos desastres naturales se incrementen; por tanto, cobran un papel protagónico las medidas preventivas a corto, mediano y largo plazo. 


\section{REFERENCIAS BIBLIOGRÁFICAS}

1. Ministerio de Salud. Vamos a prevenir. Plan de comunicación: FEN 2015 [Internet]. Lima: Ministerio de Salud; 2015 [citado el 23 de abril del 2017]. Disponible en:

http://www.minsa.gob.pe/portada/especiales/2015/fenomeno/ index.asp?op=3

2. Gomez R. Catacaos contra el dengue. Lima: Diario La Republica; 23 de abril del 2017.

3. Huarcaya E, Leyva R, Llanos A. Influencia de factores climáticos sobre las enfermedades infecciosas. Rev Med Hered. 2004;15(4):218-24.

4. Consejo Nacional del Ambiente. Perú: vulnerabilidad frente al cambio climático. Lima. Manatí Gráfico S.A; 1999.

5. Felipe Ortiz de Zevallos M. El Fenómeno de El Niño y el Clima. En: El Perú en los albores del siglo XXI. Ciclo de Conferencias 1997-1998. Lima, Perú: Ediciones del Congreso del Perú; 1998. p. 201-42.

6. Servicio Nacional de Meteorología e Hidrología. Estudio del Fenómeno El Niño 1997/98. Lima: Servicio Nacional de Meteorología e Hidrología; 1999. p.125.
7. Bravo W, Bravo F. El efecto del fenómeno El Niño en las enfermedades dermatológicas. Fol Dermatol Per. 2001; 12: 29-36.

8. Laura M, Loli O, Querevalu A, Rojas A, Ramos D. Dermatomicosis en las Áreas rurales del norte del litoral peruano Durante el Fenómeno. Dermatología Peruana. 1998; Suplemento especial: 6o. (Citado el 23 de abril del 2017) Disponible en: http://sisbib.unmsm.edu.pe/BVRevistas/ dermatologia/es_set1998/trabajoscarteloz.htm\#TC-26

9. Esteva $L$, Vargas C. A model of dengue disease with variable human population. J Math Biol. 1999; 38: 220- 240.

10. Brown L, Murray V. Examining the relationship between infectious diseases and flooding in Europe. Disaster Health. 2013; 1(2): 117-127.

11. Jafari N, Shahsanai A, Memarzadeh M, Loghmani A. Prevention of communicable diseases after disaster. J Res Med Sci. 2011; 16(7): 956-962.

12. Greaney P, Pfiffner S, David Wilson D, editors. Humanitarian Charter and Minimum Standards in Humanitarian Response. Southampton, United Kingdom: The Sphere Project; 2011.

\section{Ahora puede enviar sus artículos para Acta Médica Peruana en nuestro Open Journal System:}

\section{www.amp.cmp.org.pe}

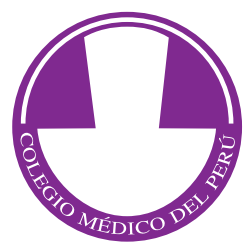

